\title{
EMC effect in the Drell-Yan process at COMPASS
}

\author{
Evgenii Mitrofanov (On behalf of COMPASS Collaboration) $)^{1, \star}$ \\ ${ }^{1}$ Joint Institute for Nuclear Research, Dubna
}

\begin{abstract}
.
The EMC effect or a modification of parton distributions in bound nucleons as compared to free ones, has been extensively studied during the last 30 years but its full understanding is still lacking. The COMPASS experiment at CERN will provide new results on the EMC effect, originating from the Drell-Yan process and studied in the $190 \mathrm{GeV} / c \pi^{-}$ beam scattering on the ammonia and tungsten targets. The present understanding of the EMC effect and experimental possibilities of COMPASS in this context are discussed.
\end{abstract}

\section{Introduction}

The momentum distribution functions of the partons within the hadron $\left(q\left(x, Q^{2}\right), \bar{q}\left(x, Q^{2}\right), g\left(x, Q^{2}\right)\right.$ ), called Parton Distribution Functions (PDFs) describe the internal structure of hadrons in the quarkparton model. They represent the probability densities to find a parton carrying a proton momentum fraction $x$ at a negative four-momentum transfer squared $Q^{2}$. At the leading order in QCD the PDFs are related to the structure function $F_{2}\left(x, Q^{2}\right)$ which is directly accessible in deep-inelastic charged lepton-nucleon scattering experiments:

$$
F_{2}\left(x, Q^{2}\right)=\sum_{q=u, d, s . .} x e_{q}^{2}\left[q\left(x, Q^{2}\right)+\bar{q}\left(x, Q^{2}\right)\right],
$$

where $e_{q}$ is the quark charge (in units of elementary charge $|e|$ ).

\section{EMC effect}

The EMC effect is a modification of quark and gluon distributions in bound nucleons by the nuclear environment. It was discovered by the European Muon Collaboration in 1983 [1] in the deep inelastic scattering of muons. The ratio of structure function $F_{2}$ per nucleon for iron and deuterium targets was measured as a function of $x$. It was found to be different from unity and at least for $x \lesssim 0.7$ not following expectations from Fermi-motion calculations. This result demonstrated for the first time that the structure function $F_{2}$ is modified, when nucleons are embedded in a nucleus. Since then the EMC effect has been observed in the numerous experiments. Figure 1 shows a typical behavior of the ratio $\sigma^{A} / \sigma^{D}$ as a function of $x$.

Dependences of nuclear modifications of $F_{2}$ on kinematics and various nuclear properties like mass, density or radius of a nucleus are rather well-known, but, nevertheless, the origin of the effect

\footnotetext{
^e-mail: evgenii.mitrofanov@cern.ch
} 
is still not fully understood. Numerous explanations of the EMC effect have been proposed: nuclear binding, pion excess in nuclei, multi-quark clusters, dynamical rescaling, medium modification, shortrange correlations, etc. However, there is no generally accepted model for the effect over all $A$ and $x$. Comprehensive reviews of the EMC effect can be found in Refs. [5, 12-15].

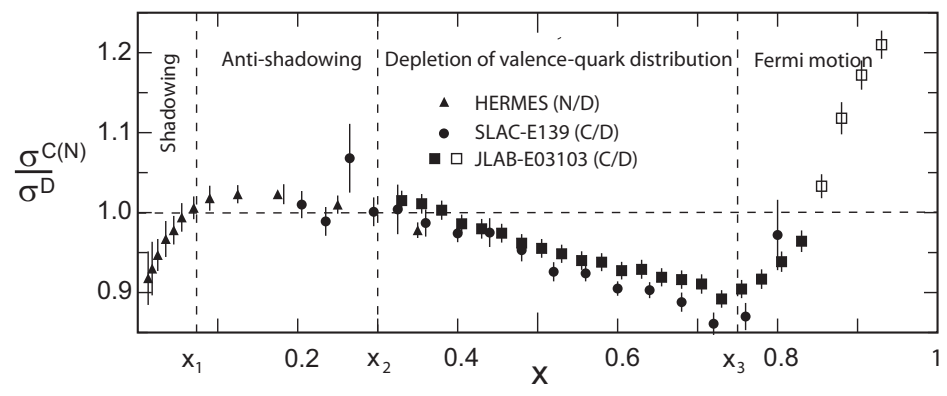

Figure 1. Ratio $\sigma^{C(N)} / \sigma^{D}$ as a function of $x$ from HERMES [2], SLAC-E139 [3], and JLAB-E03103 [4]. Figure comes from [5].

\section{EMC effect in the Drell-Yan process}

Apart of numerous deep inelastic experiments, the EMC effect has also been experimentally verified in the time-like region using both the pion- and the proton-induced Drell-Yan reactions in the E772 [6], E866 [7], NA3 [8], and NA10 [9] experiments. In the Drell-Yan process a quark (anti-quark) with the momentum fraction $x_{1}$ from the beam hadron and an anti-quark (quark) of the target nucleon with the momentum fraction $x_{2}$ annihilate via a virtual photon (contribution of the Z-boson exchange at the discussed energies is extremely small) into a charged-lepton pair: $q\left(x_{1}\right) \bar{q}\left(x_{2}\right) \rightarrow \gamma^{*} \rightarrow l^{+} l^{-}$. The Drell-Yan cross section is

$$
\frac{d^{2} \sigma_{D Y}}{d x_{1} d x_{2}}=K \frac{4 \pi \alpha^{2}}{9 x_{1} x_{2}} \frac{1}{s} \sum_{q=u, d, s \ldots} e_{q}^{2}\left[q\left(x_{1}\right) \bar{q}\left(x_{2}\right)+\bar{q}\left(x_{1}\right) q\left(x_{2}\right)\right],
$$

where $K \approx 2$ is a factor representing the deviation from the simple parton model due to QCD corrections, $s$ is the squared total energy of the $q\left(x_{1}\right) \bar{q}\left(x_{2}\right)$ system, $\alpha$ - the fine-structure constant.

The pion-induced Drell-Yan process is complementary to the DIS process and can provide another experimental tool that is sensitive to flavour-dependent effects in the nuclear quark distributions [10]. Keeping only the dominant terms in the cross section, one obtains

$$
\frac{\sigma_{D Y}^{\pi^{-} A}}{\sigma_{D Y}^{\pi^{-} D}} \approx \frac{u_{A}\left(x_{2}\right)}{4 u_{D}\left(x_{2}\right)},
$$

where $u_{A}\left(x_{2}\right)$ and $u_{D}\left(x_{2}\right)$ are PDFs for u-quark in a nucleus with a mass A and deuterium, respectively. The previous measurements of a nuclear dependence of the pion-induced Drell-Yan cross section have been performed in the NA3 [8] and NA10 [9] experiments. The ratio (3) measured by NA10 for tungsten and deuterium targets using a negative pion beam of $140 \mathrm{GeV} / c$ and $286 \mathrm{GeV} / c$, is shown in the left and right panels of Fig 2 respectively. However the accuracy is too low for detailed tests of various theoretical models. 

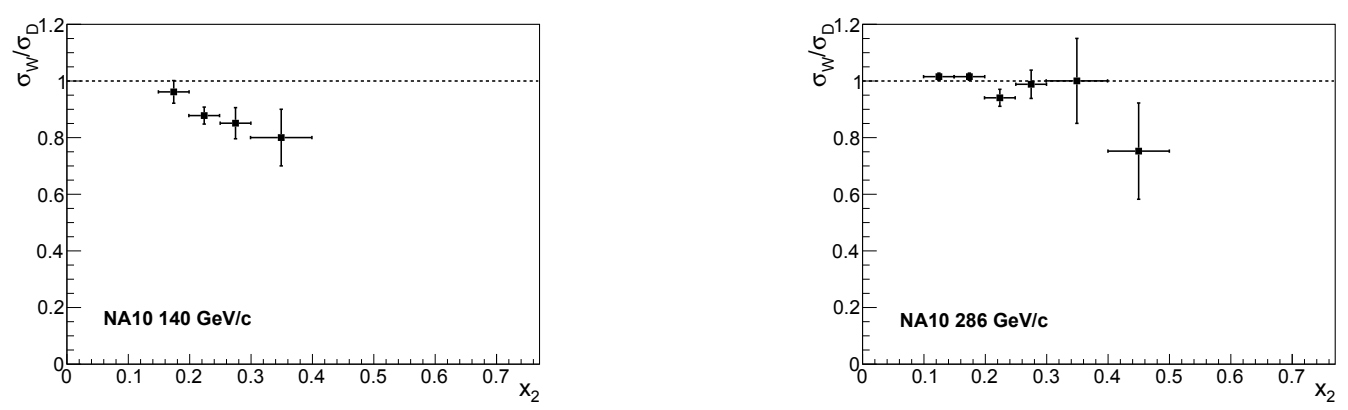

Figure 2. Ratio of the Drell-Yan cross sections for $\mathrm{W}$ and D measured by the NA10 Collaboration using a $\pi^{-}$ beam of $140 \mathrm{GeV} / c$ (left) and $286 \mathrm{GeV} / c$ (right) [9].

\section{COMPASS experiment}

COMPASS is a fixed-target experiment at a secondary beam of the Super Proton Synchrotron at CERN. The purpose of this experiment is a study of hadron structure and hadron spectroscopy with muon and hadron beams of high intensity. The experiment has an intensive physics programme which includes such topics as a study of nucleon spin structure in the semi-inclusive deep inelastic scattering and Drell-Yan process, search for new exotic hadron states in diffractive, central, Primakoff and leptoproduction, tests of the chiral theory predictions, measurement of generalized parton distributions, etc.

\subsection{COMPASS setup}

The COMPASS setup (Fig. 3) can be divided into four parts along the beam axis. It starts with the beam line telescope and the detectors that identify the incoming beam particles. It is followed by the target region, which is specific for each of the COMPASS physics programmes. The third part called the Large Angle Spectrometer, built around the first dipole magnet SM1, detects scattered particles with polar angles of up to $180 \mathrm{mrad}$. The fourth part is the Small Angle Spectrometer, which occupies the downstream part of the setup and detects particles at small angles (less than $30 \mathrm{mrad}$ ) and momenta larger than $5 \mathrm{GeV} / c$.

\subsection{EMC effect at COMPASS}

The ratio (3) of the cross sections calculated for the COMPASS kinematic coverage using the PDFs for deuterium and gold from the model of Ref. [10] is presented in Fig. 4 as a function of $x_{2}$ for the fixed mass $M_{l^{+} l^{-}}=5 \mathrm{GeV} / c^{2}$ (left) and as a function of $M_{l^{+} l^{-}}$for the fixed value $x_{1}=0.5$.

Data taking for the Drell-Yan process study with a negative pion beam of $190 \mathrm{GeV} / \mathrm{c}$ was performed at COMPASS in 2014 and 2015. The main ammonia target, a tungsten beam plug of the hadron absorber and an additional aluminium target present in the setup can be used to study the EMC effect. The observed dimuon mass spectrum and the kinematic range of $x_{1}$ and $x_{2}$ for $M_{\mu \mu}>4$ $\mathrm{GeV} / c^{2}$ covered by COMPASS for the ammonia target are shown in the left and right panels of Fig. 5 , respectively. Dimuon mass resolution for $J / \psi$ peak is about $0.2 \mathrm{MeV} / c^{2}$ for the ammonia, while for the tungsten plug it is a bit worse. Thus only the mass range above $5 \mathrm{GeV} / c^{2}$ can be used for investigation of the EMC effect, which defines a lower limit of $x_{2}$ range accessible by COMPASS to be 0.07 . 

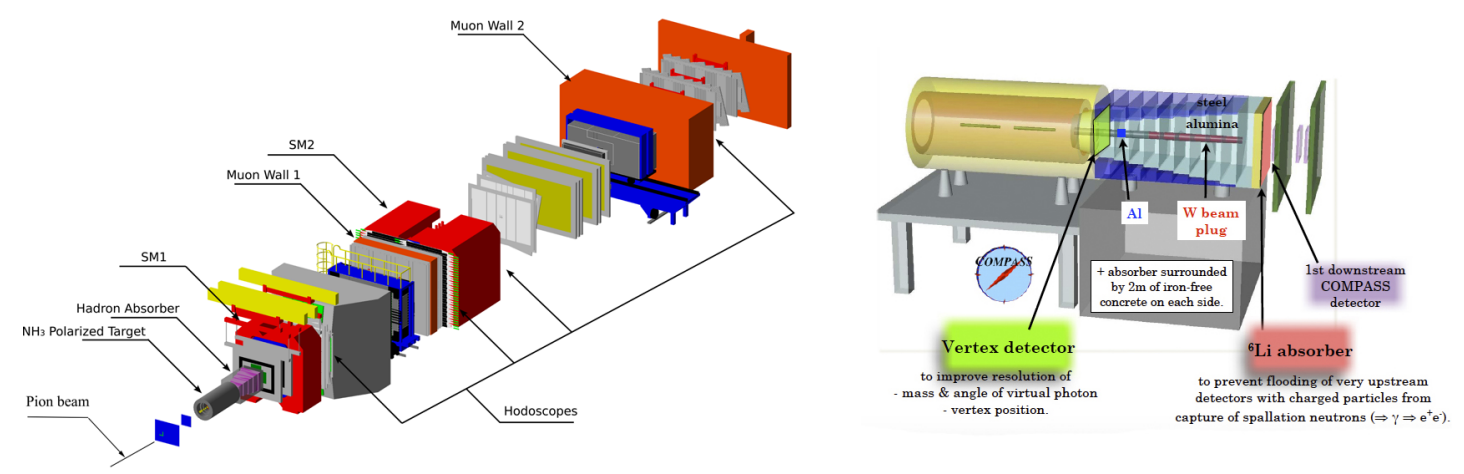

Figure 3. Left: sketch of the COMPASS setup for data taking with pion beam. Right: the ammonia target region.
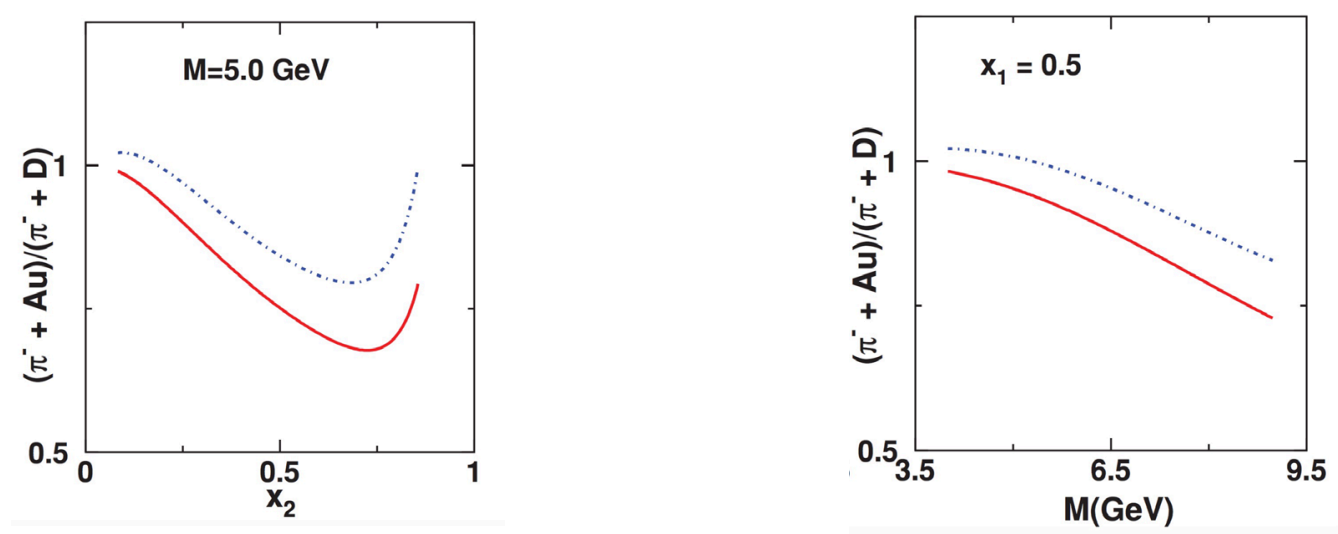

Figure 4. Ratio of the Drell-Yan cross sections for Au and D calculated for the COMPASS kinematic coverage using the PDFs from the model of Ref. [10] as a function of $x_{2}$ for the fixed mass $M_{l^{+} l^{-}}=5 \mathrm{GeV} / c^{2}$ (left) and as a function of $M_{l^{+} l^{-}}$for the fixed value $x_{1}=0.5$.
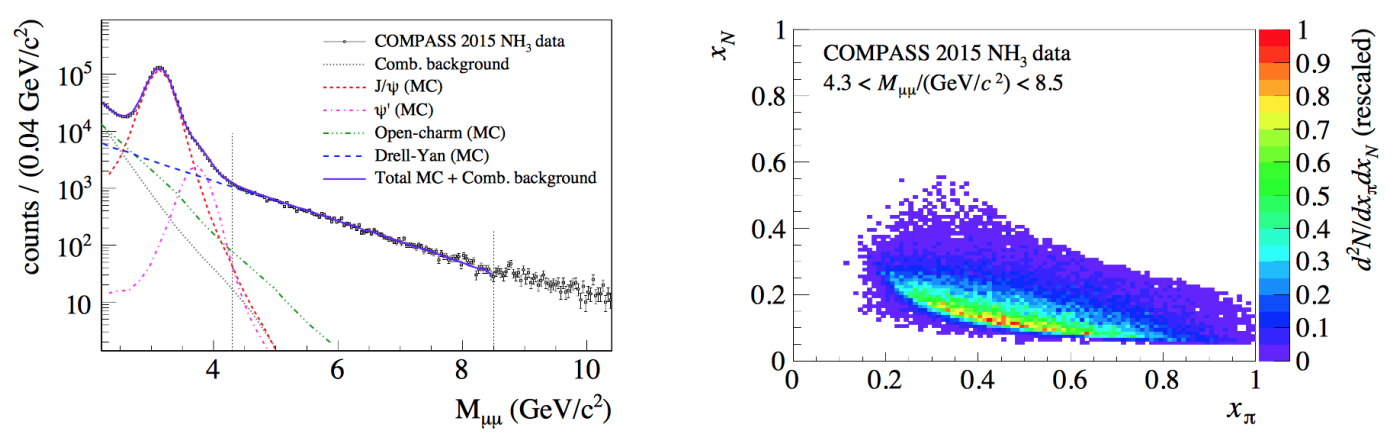

Figure 5. Dimuon mass spectrum (left) and kinematic range of $x_{1}$ and $x_{2}$ for $M_{\mu \mu}>4 \mathrm{GeV} / c^{2}$ covered by COMPASS (right) for the ammonia target [11]. 


\section{Summary}

After 30 years the origin of the EMC effect is still not fully understood and there is no no model which describes the whole range of $x$. An experimental tool that is sensitive to flavour-dependent effects in the nuclear quark distributions is the pion-induced Drell-Yan process which allows to measure EMC effect separetly for $\mathrm{u}$ and $\mathrm{d}-$ quarks.

The COMPASS experiment at CERN will provide new results on the EMC effect originating from the Drell-Yan process $\pi^{-}+A \rightarrow \mu^{+}+\mu^{-}+X$ (where $A$ is proton, tungsten and aluminium) covering values of $x$ down to 0.07 , i.e. lower than in other experiments

\section{References}

[1] J. J. Aubert et al. (EMC Collaboration), Phys. Lett. B 123, 275 (1983).

[2] A. Airapetian et al. (HERMES Collaboration), Phys. Lett. B 567, 339 (2003).

[3] J. Gomez et al. (SLAC-E139 Collaboration), Phys. Rev. D 49, 4348 (1994).

[4] J. Seely et al. (JLAB-E03103 Collaboration), Phys. Rev. Lett. 103, 202301 (2009).

[5] K. Rith, Subnucl. Ser. 51, 431 (2015).

[6] D. M. Alde et al. (FNAL-E772 Collaboration), Phys. Rev. Lett. 64, 2479 (1990).

[7] M. A. Vasiliev et al. (FNAL-E866 Collaboration), Phys. Rev. Lett. 83, 2304 (1999).

[8] J. Badier et al. (NA3 Collaboration), Phys. Lett. B. 104, 335 (1981).

[9] P. Bordalo et al. (NA10 Collaboration), Phys. Lett. B. 193, 368 (1987).

[10] D. Dutta et al., Phys. Rev. C. 83, 042201 (2011).

[11] M. Aghasyan et al. (COMPASS Collaboration), Phys. Rev. Lett. 119, 112002 (2017).

[12] M. Arneodo, Phys. Rep. 240, 301 (1994).

[13] D. Geesaman, K. Saito, A. W. Thomas, Ann. Rev. Nucl. Part. Sci. 45, 337 (1995).

[14] P.R. Norton, Rep. Prog. Phys. 66, 1253 (2006).

[15] S. Malace et al., Int. J. Mod. Phys. E. 23, 1430013 (2014). 